\title{
Real-Time Error Recovery Utilizing Layered Media Multicast Control (LMMC)
}

\author{
Homayoun Yousefi'zadeh Hamid Jafarkhani \\ Electrical Engineering and Computer Science Department \\ University of California, Irvine \\ [hyousefi, hamidj] @uci.edu
}

\begin{abstract}
In this paper, we present an optimal solution to the problem of error control in layered and replicated media systems satisfying real-time delay constraints. In doing so, we rely on an apriori estimate of loss along with a hybrid proactive FECARQ scheme to statistically guarantee the quality of service for receivers. Our optimal Layered Media Multicast Control (LMMC) solution to a formulation of the error control problem analytically determines the redundancy assignment of individual groups associated with a layered media system minimizing a cost metric defined over wasted bandwidth of redundancy.

Index Terms-Multicast IP Networks, Layered Media, Replicated Media, Error Control, Apriori Estimate of Loss, Statistical Guarantee of QoS, FEC, ARQ, Optimal Iterative Partitioning.
\end{abstract}

\section{INTRODUCTION}

Transmitting real-time compressed digital media over multicast IP networks has been the subject of heavy research in the recent years as surveyed by $\mathrm{Li}$ et al. in [6] and the references cited therein. Replicated media streams approach first presented by Cheung et al. [2] within the context of DSG protocol and layered media streams approach first proposed by McCanne et al. [9] in the context of RLM protocol following the work of Deering et al. [4] in the context of multicast routing are convincingly the two most important methods in this area.

Real-time video and audio have limited tolerance for random loss within the compressed digital stream. The quality of decoded media at a receiver is subject to significant degradation as the result of excessive loss from network congestion or latency. In order to overcome the loss effects, error control techniques can be used. There have been three general error control approaches in the context of multicasting. In Retransmissionbased Automatic Repeat reQuest (ARQ), retransmissions occur only if data can be delivered before the real-time deadline. Two of such approaches are the error control aspect of LVMR presented by Li et al. [7] and STORM presented by Xu et al. [15]. In Forward Error Correction (FEC), the source assigns a portion of its bandwidth for proactive transmission of repair packets to the receivers. Among the rich set of articles in the literature, the two most closely related to our work are by Rubenstein et al. [12] in which the idea of using real-time reliable multicast using proactive FEC is proposed and Rhee et al. [11] in which a proactive FEC reliable multicast layering scheme is presented. There are also hybrid FEC-ARQ approaches suggesting different alternatives for proactive transmission of redundant packets based on retransmission requests. Chande et al. [1] and Chou et al. [3] proposed hybrid error control systems by augmenting layered FEC with a pseudo-layered ARQ system. Towsley et al. [13] and Nonnenmacher et al. [10] respectively analyzed the advantages of hybrid approaches over a stand-alone ARQ and in conjunction with local recovery.

The objective of this article is to provide an analytical framework for the error control of layered and replicated media systems over multicast IP networks. The scope of our work addresses the issues of media systems' error control and consequently QoS within the context of Layered Media Multicast Control (LMMC) protocol. In this study, we assume the existence of congestion and flow control mechanisms capable of dynamically addressing inter-session fairness and flow control issues. A closely related flow control example is given in [16]. Anther related example is given in [14]. Rather, the error control aspect of LMMC manifests in dynamic distribution of an available bandwidth among data and redundant traffic portions. For each individual multicast group related to a layered or a replicated media system, LMMC specifies the assignment of data and redundancy bandwidths such that the resulting bandwidth wastage of redundancy is minimized. The main contribution of this paper is in the following areas. First, the paper proposes a method allowing individual receivers of each multicast group to provide the source with an apriori estimate of their redundancy requirement in order to statistically guarantee quality of service (QoS). Second, the paper formulates an optimal control problem aiming at minimization of the wasted bandwidth considering specific constraints of real-time latency and the impact of feedback implosion. The paper also provides a low complexity analytical solution to the formulation of the problem. It is important to note that the technique proposed in this paper can be independently applied to both replicated and layered media systems.

An outline of the paper follows. In Section II, we provide an analysis of loss relying on a temporally correlated model in order to provide a statistical guarantee of $\mathrm{QoS}$ for the receivers of a media system. In Section III, we propose an analytical solution to a formulation of the optimal error control problem of media systems defined over a cost function indicating the wasted bandwidth of a media session. Specific formulation of this section also eliminates the impact of feedback implosion. In Section IV, we focus on performance evaluation and provide simulation results along with practical considerations. Finally, Section VII concludes the paper.

\section{LMMC ANALYSIS OF REDUNDANCY}

In this section, we focus on the analysis of redundancy for a replicated and/or layered layered media system. Such a system was studied in our LMMC rate allocation and partitioning work of [17]. Consistent with our previous work, we con- 
sider a media session with an ordered partitioning of $N$ receivers into $K$ data groups $\left\{G_{1}|\cdots| G_{K}\right\}$ and group data rates of $g_{1}, g_{2}, \cdots, g_{K}$ such that $g_{1} \leq g_{2} \leq \cdots \leq g_{K}$.

We start by adopting the general notion of round-based delivery of real-time multicast information for LMMC error control scheme as proposed in [12]. A round-based hybrid FEC-ARQ error recovery scheme for delivering multicast information appropriately applies to real-time scenarios in which a hard deadline has to be met. This deadline typically has to do with the availability of data at the playback time in a multimedia application. For each receiver, a hard deadline can be expressed in terms of the available number of rounds. Assuming that a hard deadline is given by $\tau_{k}$ time units for a data group $G_{k}$ and a receiver $i$ in data group $G_{k}$ measures the average round trip time of a packet from the session source to be $R T T_{i}$ time unit, the number of available rounds for receiver $i$ is calculated as

$$
R D_{i}=\left\lfloor\frac{\tau_{k}}{R T T_{i}}\right\rfloor
$$

The available number of rounds for data group $G_{k}$ of a media session is, then, defined as

$$
\Gamma_{k}=\min _{i} R D_{i} \quad \forall i \in G_{k}
$$

Considering the fact that the proposed protocol of [12] requires a receiver to specify the number of packets going to some intermediate round possibly the last round, our important observation is that the proposed protocol of [12] is not quite appropriate for error recovery techniques relying on an apriori knowledge or estimate of loss. Taking into consideration specific design issues of LMMC pertaining to integration of its rate allocation/partitioning aspects with its error control aspect, we hence propose a new way of providing a statistical guarantee relying on an apriori estimate of loss.

As pointed out in many research articles, Internet packet loss typically undergoes burst loss representing temporally correlated loss. This is related to the fact that many of the routers utilized in the Internet have deployed drop-tail routing. The twostate Gilbert model provides an elegant mathematical model to capture the loss behavior of real networks. In [18], we introduce a closed form solution for the probability distribution $D(u, v)$ indicating the number of received packets $v$ from the number of transmitted packets $u$ under the Gilbert loss model. In our method of requesting redundant packets, we propose that an individual receiver $i$ of a media session data group $G_{k}$ relies on the closed form probability distribution $D(u, v)$ for the Gilbert loss model given in [18] to provide a per round apriori estimate of loss. In our analysis, we consider a block recovery probability of $\Pi_{k}$ with equal per round probabilities of $\pi_{k}$ for the available number of rounds in data group $G_{k}$ of a media session. Assuming the source of a media session only initiates a new transmission round for the receivers of data group $G_{k}$ as the result of receiving at least one NAK from the receivers of the group, we relate the two quantities as

$$
\Pi_{k}=1-\left(1-\pi_{k}\right)^{\Gamma_{k}}
$$

yielding

$$
\pi_{k}=1-\sqrt[\Gamma_{k}]{1-\Pi_{k}}
$$

Hence, given the overall probability of block recovery $\Pi_{k}$ for data group $G_{k}$, the per round probability of block recovery is calculated from Equation (4). In this scheme, a receiver $i$ obtains an estimate of required redundant packets by calculating the expected number of received packets according to its probability distribution $D\left(u_{i}, v_{i}\right)$ going from one round to another ${ }^{1}$. More precisely, for a given block size $B_{k}$ of the data group $G_{k}$ and starting from an initial value of the required packets $v_{i}^{1}=(\psi-1) B_{k}$ for receiver $i$ in the first round where $1 \leq \psi<2$, the number of requested packets $u_{i}^{j}$ in round $j$ is calculated based on $v_{i}^{j}$ the number of packets still required by receiver $i$ in round $j$. The value of $v_{i}^{j}$ is calculated by deducting the expected number of arrived packets in the previous rounds from $(\psi-1) B_{k}$. The overall required redundancy of receiver $i$ is, then, calculated as

$$
r_{i}=\min \left(\sum_{j=1}^{\Gamma_{k}} u_{i}^{j}, B_{k}\right)
$$

A receiver $i$ can announce its overall redundancy $r_{i}$ and per round required redundancy sequence $\left\{u_{i}^{1}, \cdots, u_{i}^{\Gamma_{k}}\right\}$ to the media source. A receiver can utilize the iterative algorithm of [18] to obtain its required redundancy with a time complexity of $\mathcal{O}\left(z B_{k}\right)$ for a calculated number $z \leq B_{k}$. We note that the complexity of such an algorithm is lower than that of a dynamic programming algorithm $\mathcal{O}\left(B_{k}^{2}\right)$.

At the end of this section, we note that LMMC eliminates the impact of feedback implosion from the discovery process of the receivers by forcing the receivers to report their redundancy requirements utilizing a randomly set timer-based scheme as the one discussed in [13].

\section{Optimal LMMC ERROR CONTROL PROTOCOL}

Having calculated the required redundancy for individual receivers of a media multicast group, we now focus on the formulation of the optimal error control problem and LMMC's analytical solution to the problem. We formulate our layered real-time error control problem in a way similar to Layered Multicast Recovery (LMR) protocol proposed in [11]. However, we make note of the differences in the formulation as well as the solution. We note that unlike the formulation of [11] that is intended for reliable multicast, the formulation of our problem is within the context of layered or replicated media systems and is hence subject to real-time constraints applied to media systems. We also note that the formulation of the error control problem for reliable multicast systems is subject to feedback implosion where as our formulation is capable of effectively eliminating the impact of feedback implosion. In addition, because of targeting at providing a set of integrated protocols for media systems, we rely on an apriori estimate of redundancy. Finally rather than relying on dynamic programming, we propose a lower complexity analytical solution to the problem within the context of LMMC error control protocol.

\footnotetext{
${ }^{1}$ We provide a discussion of dynamically requesting redundant packets instead of relying on an estimate of redundancy that is applicable to moderate size multicast groups in [19].
} 
Due to space limitation, we only describe the highlights of LMMC error control protocol in this paper. Briefly, we note that LMMC error control protocol calls for adopting an iterative process when solving integrated rate allocation, partitioning, and error control problems in order for the source of a media system to dynamically adapt to redundancy requirement of a media session. In each iteration also referred to as a polling period, the source first detects the number of available rounds in a data group $G_{k}$ followed by polling the receivers for their required redundancy. Each receiver then estimates its redundancy requirement and reports it back to the source. Going from one round to another, only one of the receivers not capable of recovering the data block with size $B_{k}$ multicasts a NAK message to the group notifying the source about the need for initiating the next round. The proposed mechanism effectively eliminates the NAK traffic as the overall number of transmitted NAKs is in the order of number of rounds $\Gamma_{k}$. We refer the reader to [19] for formal description of LMMC error control protocol as well as integration process of LMMC error control protocol with LMMC rate allocation and partitioning protocol.

In our error control model for media systems, we associate $\varsigma_{k}$ redundant multicast groups with every individual data group $G_{k}$. Although we apply a fixed value to the parameter $\varsigma_{k}$ in our formulation, we mention that the choice of $\varsigma_{k}$ is a design parameter with the objective of providing a balance between bandwidth wastage and overhead of managing multicast groups. Assuming a block size of $B_{k}$ for data group $G_{k}$, the source transmits $B_{k}$ data packets to data group $G_{k}$ followed by $\rho_{\varsigma_{k}}$ redundant packets on $\varsigma_{k}$ independent redundant groups. From a layering stand point, the formulation of the error control problem is similar to the two-phase rate allocation and partitioning problem of our earlier work in [17]. This means that a receiver can subscribe to a redundant group only if it has already subscribed to all of the previous redundant groups. However, we note that in this case the collection of redundancy groups $\left\{1, \cdots, \varsigma_{k}\right\}$ combined together are considered the redundancy groups associated with data group $G_{k}$ in the rate allocation and partitioning problem.

In this analysis, we consider a partitioning of the receivers of data group $G_{k}$ into $\varsigma_{k}$ groups according to their redundancy requirement. For data group $G_{k}$ with $N_{k}$ receivers, we associate $\varsigma_{k}$ redundancy groups each redundancy group carrying a portion of redundant traffic. For a partitioning $\Omega_{k}=$ $\left\{R_{1}|\cdots| R_{\varsigma_{k}}\right\}$ of data group $G_{k}=\left\{1, \cdots, N_{k}\right\}$ with ordered group redundancy rates of $\rho_{1}, \rho_{2}, \cdots, \rho_{\varsigma_{k}}$ such that $\rho_{1} \leq \rho_{2} \leq$ $\cdots \leq \rho_{\varsigma_{k}}$, the layer redundancy rates of a layered error control scheme are calculated in the form of

$$
\rho_{1}, \rho_{2}-\rho_{1}, \rho_{3}-\rho_{2}, \cdots, \rho_{\varsigma_{k}}-\rho_{\varsigma_{k}-1}
$$

A receiver in redundancy group $j$ subscribes to layers 1 through $j$ receiving an aggregated redundancy rate of $\rho_{j}$. If required, LMMC error control protocol allows receivers to subscribe to extra redundancy groups only at the beginning of each polling period. This is necessary to control the overhead of multicast group joins and leaves considering real-time constraints of media systems.

In order to formulate a per group error control problem for individual data groups $G_{k}$ with $k \in\{1, \cdots, K\}$ of a me- dia session while considering the impact of feedback implosion, we observe that for a block size of $B_{k}$ in group $G_{k}$ with $k \in\{1, \cdots, K\}$, all of the receivers' reported redundancy numbers are in the range of $\left[1, B_{k}\right]$. The source can, hence, rely on a hierarchical tree-based feedback aggregation protocol similar to the one proposed in [8] or [5] to identify the subgroup of receivers with redundancy requirements matching $i$ redundant packets in the range $\left[1, B_{k}\right]$. By sending individual polling packets sweeping the redundancy range $\left[1, B_{k}\right]$, the source can effectively eliminate the impact of feedback implosion. Assuming there exists a per data group upper bound on the maximum number of redundant packets in the form of $\max _{i} r_{i}=U_{k}$ where $U_{k} \leq B_{k}$, we formulate the optimal error control problem of data group $G_{k}$ of a media session as

$$
\begin{aligned}
\min _{\rho_{1}, \cdots, \rho_{\varsigma_{k}}} E C W_{k} & =\min _{\rho_{1}, \cdots, \rho_{\varsigma_{k}}} \sum_{j=1}^{\varsigma_{k}} \sum_{i=1}^{B_{k}} w_{j i}\left(\rho_{j}-i\right) \\
\text { Subject To: } & \rho_{\varsigma_{k}} \leq B_{k}
\end{aligned}
$$

where $\varsigma_{k}$ with $k \in\{1, \cdots, K\}$ is the number of redundant groups associated with data group $G_{k}$, the function $E C W_{k}$ is the bandwidth wastage of data group $G_{k}$ over all of its redundancy groups $R_{j}$ with $j \in\left\{1, \cdots, \varsigma_{k}\right\}$, and $w_{j i}$ is the weighting function associated with the number of receivers requesting redundancy $i$ where $\sum_{j=1}^{\varsigma_{k}} \sum_{i=1}^{B_{k}} w_{j i}=N_{k}$. It is also important to note that $w_{j i}=0$ if $\rho_{j}<i$ or if a receiver does not belong to redundancy group $j$. Rather than relying on a dynamic programming approach as suggested in [11], we utilize an analytical approach in solving Equation (7) with Constraint (8). In our approach, we introduce an iterative partitioning scheme that is guaranteed to converge to a local minimum. In our partitioning strategy, it is imperative to assign a receiver $i$ with required redundancy $r_{i}$ to the redundancy group $R_{j}$ with the group redundancy rate $\rho_{j}$ for a set of given group redundancy rates $\left\{\rho_{1}, \cdots, \rho \varsigma_{k}\right\}$, if the receiver bandwidth wastage $\left(\rho_{j}-r_{i}\right) \geq 0$ is minimized for the choice of $\rho_{j}$. As the result, we make the observation that the optimal receiver partitioning strategy has to assign receiver $i$ with the redundancy rate $r_{i}$ to the redundancy group $R_{j}$ with the group redundancy rate $\rho_{j}$ such that

$$
0 \leq\left(\rho_{j}-r_{i}\right) \leq\left(\rho_{l}-r_{i}\right) \quad l \in\left\{1, \cdots, \varsigma_{k}\right\}
$$

It is proven in Lemma (II.1) of [11] that for such a partitioning of the receivers utilized in LMMC formulation, the optimal redundancy rate of each partition is equal to the largest redundancy requirement of the receivers of that specific partition, i.e.,

$$
\rho_{j}^{*}=\max _{i \in R_{j}} r_{i} \quad j \in\left\{1, \cdots, \varsigma_{k}\right\}
$$

Let us now pay attention to the implication of the latter result in case of applying an optimal partitioning strategy to a simple partitioning of the receivers into two redundancy groups. For an ordered partitioning $\Omega_{k}=\left\{R_{1} \mid R_{2}\right\}$ of the receivers $G_{k}=$ $\left\{1, \cdots, L_{1}, L_{1}+1, \cdots, L_{2}\right\}$ with $L_{1}$ indicating the last receiver of partition $R_{1}$ and $L_{2}$ indicating the last receiver of partition $R_{2}$, we note that a receiver $s$ with redundancy requirement $r_{s}$ and all of the receivers with greater redundancy requirements in partition $R_{1}$ have to move to partition $R_{2}$ if

$$
L_{1}\left(r_{L_{2}}-r_{L_{1}}\right)<(s-1)\left(r_{L_{2}}-r_{s-1}\right)
$$


Likewise, a receiver $t$ with redundancy requirement $r_{t}$ and all of the receivers with lower redundancy requirements in partition $R_{2}$ have to move to partition $R_{1}$ if

$$
L_{1}\left(r_{L_{2}}-r_{L_{1}}\right)<t\left(r_{L_{2}}-r_{t}\right)
$$

Generalizing these results for an ordered partitioning $\left\{R_{1}|\cdots| R_{\varsigma_{k}}\right\}$ of the receivers, we propose the following iterative algorithm to solve the optimal error control problem of Equation (7) with Constraint (8).

\section{LMMC Error Control Algorithm: An Iterative Lay- ered Partitioning Approach}

- Step 1: Start from an initial ordered partitioning of the receivers by uniformly distributing the receivers among the redundancy groups. In addition, set the initial iteration number $i t=0$ and the maximum number of iterations it $t_{\max }$.

- Step 2: Calculate the optimal redundancy rates of each partition $R_{j}$ with $j \in\left\{1, \cdots, \varsigma_{k}\right\}$ from Equation (10) and the resulting error control cost function $E C W_{k}$ from Equation (7). Save the previously calculated $E C W_{k}$ in variable $q_{1}$ and the currently calculated $E C W_{k}$ in variable $q_{2}$.

- Step 3: If $\frac{\left|q_{1}-q_{2}\right|}{q_{1}}<\delta$ or $i t>i t_{\max }$ STOP.

- Step 4: for $\left(j=\varsigma_{k}\right.$ downto 2) \{

- Repartition groups $j-1$ and $j$ according to Equation (11) and Equation (12).

\} $/ *$ for $\left(j=\varsigma_{k}\right.$ downto 2$) * /$

- Step 5: Go back to Step 2.

We note that LMMC error control algorithm moves multiple receivers with the same redundancy requirements from one redundancy group to another together. We also note that the time complexity of implementing LMMC error control algorithm is $\mathcal{O}\left(I B_{k}\right)$ where $I$ indicates the number of iterations.

Theorem 3.1: "LMMC Error Control Algorithm" mentioned in this section converges to a local minimum.

A formal proof is given in [19]. We argue that LMMC error control protocol is a practical alternative for real-time media systems from the stand point of the latency observed in multicast group join/leave operations due to the following reasons. First, the calculation of the bandwidth for individual redundant groups is done considering redundancy requirements of individual receivers. Second, the built-in polling mechanism of LMMC is responsible for adjusting the number of redundant packets according to the current loss condition of individual receivers so that the receivers do not have to subscribe to extra redundancy groups often. In addition, dropping redundant groups as the result of having a reduced loss rate does not entitle a receiver to extra latency.

\section{NumERICAL ANALYSIS}

In this section, we present the numerical results of applying LMMC error control algorithm to sample data groups in layered media scenarios. In our simulations of media systems error control, we compare LMMC results with the results of optimal LMR (OLMR) algorithm of [11] utilizing dynamic programming and heuristic LMR (HLMR) algorithm of [11] utilizing uniform distribution of redundancy. In our comparisons, we review the performance of the approaches from the stand point of tracking minimum value of the bandwidth wastage, time complexity indicated by experiment runtime, and space complexity indicated by experiment memory allocation. Additionally, we review the scalability of the techniques by covering a relatively broad range of multicast group sizes ranging from hundreds to thousands of receivers. We remind that for a block size $B$, number of iterations $I$, and number of redundancy groups $\varsigma$, the per group time complexity of LMMC error control algorithm is $\mathcal{O}(I B)$ and that of OLMR algorithm is $\mathcal{O}\left(\varsigma B^{2}\right)$. In addition, the space complexity of LMMC and OLMR error control algorithms in our implementation are $\mathcal{O}(B)$ and $\mathcal{O}\left(B^{2}\right)$ respectively assuming block size $B$ indicates an upper bound on the maximum required redundancy.

Fig. (1) and Fig. (2) compare sample results of LMMC algorithm with those of OLMR and HLMR algorithms. In each experiment, we have relied on a normal random number generator simulating receiver redundancy requirements within the range of $[1 \%, 30 \%]$. This is consistent with real network traces reported in [11]. Different figures have been obtained for different choices of block size $B$ and number of redundancy groups $\varsigma$ associated with a data group. The $\mathrm{x}$-axis of each curve is always in logarithmic scale indicating different values for the group size $N$ from the set $\{100,300,1000,3000,10000,30000,100000\}$. Each figure consists of a pair of three curves. The first set of

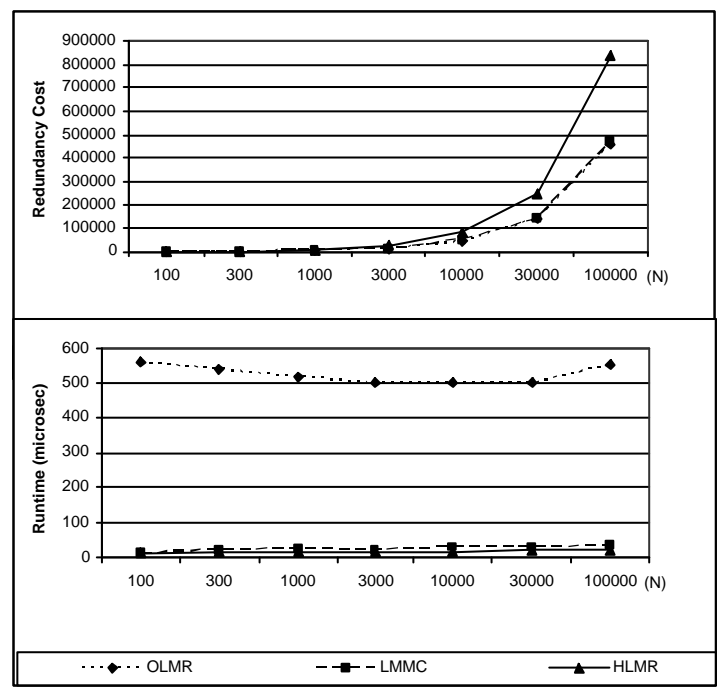

Fig. 1. Redundancy cost and runtime comparison of LMMC, OLMR, and HLMR methods vs number of receivers $(\mathrm{N})$ for $\varsigma=3$ and block size of $B=128$.

curves compare the bandwidth wastage or redundancy cost of the three techniques. While LMMC and OLMR keep a close bandwidth wastage across the board, we observe that for group sizes of 1000 or more the bandwidth wastage of HLMR departs from the other two. Considering the results, we note that HLMR can be effectively used only if the distribution of the redundancy is not highly skewed and the group size is not very large. Additionally for about 5000 experiments made by us, 


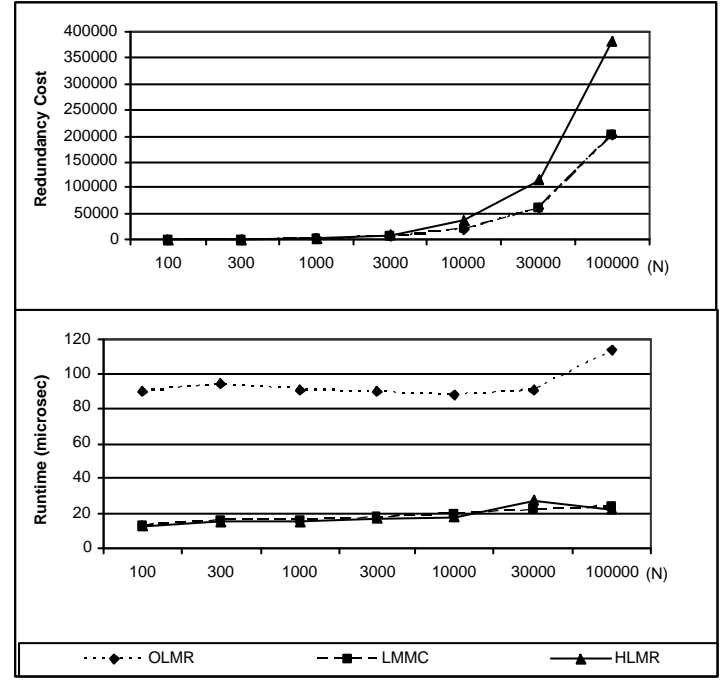

Fig. 2. Redundancy cost and runtime comparison of LMMC, OLMR, and HLMR methods vs number of receivers $(\mathrm{N})$ for $\varsigma=2$ and block size of $B=64$.

we observed a maximum $5 \%$ cost advantage of OLMR over LMMC. Considering the fact that a dynamic programming approach identifies a global optimum where as a gradient-based approach identifies a local optimum, our experiments indicate impressive convergent behavior of LMMC. The second pair of curves display the runtime of the experiments as an indicator of the time complexity of the three techniques. To our expectation, the complexity of HLMR for a small size group is the lowest among the three considering its negligible overhead of computation. In this area, a review of the results reveals closeness of LMMC results to those of HLMR and a great performance advantage of over OLMR. This is consistent with the time complexity analysis anticipating a linear dependency and a quadratic dependency on the value of $B$ in the runtime of LMMC and OLMR respectively.

We also note that LMMC error control protocol can be applied as a reliable multicast technique by considering reliable multicast constraints instead of real-time constraints. We note that the results of our experimentations in this area are consistent with the results reported in [11].

Finally, we need to discuss the impact of increasing the number of layers in solving the global problem of layered or replicated media systems. Considering the layering architecture of LMMC, we argue that utilizing a relatively large number of redundant multicast groups associated with each individual data group may be overhead prohibitive from the stand point of joining and leaving multicast groups and as discussed in Section III. As a cautionary step, our implementation of LMMC error control relies on utilizing up to three redundant groups associated with each individual data group.

\section{CONCLUSiON}

In this paper, we studied Layered Media Multicast Control (LMMC) solution to a formulation of the optimal error control problem for layered and replicated media systems over multicast IP networks. We assumed the existence of congestion and flow control mechanisms specifying a fair bandwidth available to a media session. We aimed at providing an analytical solution to a formulation of the problem minimizing the bandwidth wastage of individual multicast groups while effectively eliminating the impact of feedback implosion and providing a statistical guarantee for the quality of service of each receiver. Considering scalability of LMMC error control approach, we showed that the approach could be effectively adopted in different size point-to-multipoint groups. Finally, we evaluated the performance of LMMC solution and illustrated its applicability in realistic network topologies through the use of simulations.

\section{REFERENCES}

[1] V. Chande, H. Jafarkhani, N. Farvardin, "Joint Source-Channel Coding of Images for Channels with Feedback," In Proc. of IEEE Workshop on Inform. Theory, February 1998.

[2] S. Cheung, M. H. Ammar, X. Li, "On the Use of Destination Set Grouping to Improve Fairness in Multicast Video Distribution,” In Proc. of IEEE INFOCOM, March 1996.

[3] P.A. Chou, A.E. Mohr, A. Wang, S. Mehrotra, "Error Control for Receiver-Driven Layered Multicast of Audio and Video," IEEE Trans. On Multimedia, March 2001.

[4] S.E. Deering, D.R. Cheriton, "Multicast Routing in Datagram Internetworks and Extended LANs," ACM Trans. on Computer Systems, May 1990.

[5] B. Levine, D. Lavo, J.J. Garcia-Luna-Aceves, "The Case for Reliable Concurrent Multicasting Using Shared Ack Trees," In Proc. of ACM Multimedia, November 1996.

[6] X. Li, M. Ammar, S. Paul, "Video Multicast over the Internet," IEEE Network Magazine, April 1999.

[7] X. Li, S. Paul, P. Pancha, M.H. Ammar, "Layered Video Multicast with Retransmission (LVMR): Evaluation of Error Recovery," In Proc. of NOSSDAV, May 1997.

[8] J.C. Lin, S. Paul, "RMTP: A Reliable Multicast Transport Protocol," In Proc. of IEEE INFOCOM, 1996.

[9] S. McCanne, V. Jacobson, M. Vetterli, "Receiver Driven Layered Multicast," In Proc. of ACM SIGCOMM, September 1996.

[10] J. Nonnenmacher, M. Lacher, M. Jung, E.W. Biersack, G. Carle, "How Bad Is Reliable Multicast without Local Recovery?," In Proc. of IEEE INFOCOM, March 1998.

[11] I. Rhee, S.R. Joshi, M. Lee, S. Muthukrishnan, and V. Ozdemir "Layered Multicast Recovery (LMR)," Technical Report, NCSU, Computer Science Dept, TR-99-09, February 1999.

[12] D. Rubenstein, J. Kurose, D. Towsley, "A Study of Proactive Hybrid FEC/ARQ and Scalable Feedback Techniques for Reliable, Real-Time Multicast," Computer Communications Journal, March 2001.

[13] D. Towsley, J. Kurose, S. Pingali, "A Comparison of Sender-Initiated and Receiver-Initiated Reliable Multicast Protocols,' IEEE JSAC, vol. 15, April 1997.

[14] H.A. Wang, M. Schwartz, "Achieving Bounded Fairness for Multicast Traffic and TCP Traffic in the Internet," In Proc. of ACM SIGCOMM, September 1998.

[15] X.R. Xu, A. Myers, H. Zhang, R. Yavatkar, "Resilient Multicast Support For Continuous Media Applications,” In Proc. of NOSSDAV, May 1997.

[16] (*) H. Yousefi'zadeh, F. Fazel, H. Jafarkhani, "A Linear Optimization Approach for Achieving Flow Fairness in Unicast and Multicast Networks," Submitted for Publication.

[17] (*) H. Yousefi'zadeh, H. Jafarkhani, A. Habibi "Layered Media Multicast Control (LMMC): Rate Allocation and Partitioning," Submitted for Publication.

[18] (*) H. Yousefi'zadeh, H. Jafarkhani, “Analytical Modeling of Burst Loss: A Study of the Gilbert Model," Submitted for Publication.

[19] (*) H. Yousefi'zadeh, H. Jafarkhani, A. Habibi "Layered Media Multicast Control (LMMC): Error Control," Submitted for Publication. (*) Available at http://www.ece.uci.edu/ hyousefi/pub.html. 